\title{
The effect of novel distractors on performance in focused attention tasks: A cognitive-psychophysiological approach
}

\author{
NURIT GRONAU \\ Harvard Medical School, Charlestown, Massachusetts \\ EINAT SEQUERRA and ASHER COHEN \\ Hebrew University of Jerusalem, Jerusalem, Israel \\ and \\ GERSHON BEN-SHAKHAR \\ Hebrew University of Jerusalem, Jerusalem, Israel \\ and The Open University of Israel, Raanana, Israel
}

\begin{abstract}
In the present study, we examined whether or not novel stimuli affect performance in a focused attention task. Participants responded to a central target while an irrelevant distractor in the visual display was occasionally changed. In Experiment 1, both target and distractor were presented centrally within the focus of attention. In Experiment 2, a central target was presented along with an irrelevant distractor at a peripheral location, outside the focus of attention. Novel distractors were associated with longer latencies and enhanced orienting responses (as measured by skin conductance responses) only when presented at an attended location. In contrast, as is demonstrated in Experiment 3, the same peripheral novel distractors interfered with task performance when they possessed task-relevant information. These results indicate that there is a fundamental difference between novel stimuli and task-relevant stimuli. Whereas the former exert influence only within the focus of attention, the latter affect performance even when positioned in an unattended location. Our findings have important implications for the operation of visual attention.
\end{abstract}

Visual tasks often involve presentation of both target and distractor stimuli, requiring selection of the targets and filtering out of the distractors. Ample evidence suggests that visual attention mediates this selection by focusing either on a region (Posner, 1980) or on an object (Duncan, 1984) in the visual scene. However, selection by visual attention is not perfect, and numerous studies have demonstrated that distractors may affect performance even in tasks in which participants focus their attention on the target's location ahead of time (e.g., Eriksen \& Eriksen, 1974; Stroop, 1935). The boundary conditions for interference in focused attention tasks have been extensively investigated because they may shed light on the processes underlying the selective attention mechanism. One prominent example of this approach is the examination of the influence of distractors on performance when these distractors are presented either within or outside the focus of

This research was supported by the Israel Foundations Trustees. We thank Tram Neill, Jan Theeuwes, and Robert Rauschenberger for helpful comments, and Ori Cohen and Avia Munchik for their help in data collection. Correspondence concerning this article should be addressed to N. Gronau, Martinos Center for Biomedical Imaging at MGH, Harvard Medical School, 149 Thirteenth St., Room 2301, Charlestown, MA 02129 (e-mail: gronau@nmr.mgh.harvard.edu). attention. In this study, we examine whether novel stimuli that are irrelevant for the task interfere with focused attention tasks when they are positioned within and outside the main focus of attention. ${ }^{1}$

It is interesting to compare the influence of distractors appearing in attended and unattended locations because much evidence suggests that visual attention acts as a gating mechanism (see, e.g., LaBerge, 1983). That is, stimuli positioned inside the focus of attention gain access to higher level processes dealing with the task at hand. Consequently, distractors presented within the focus of attention allow one to observe whether or not they are sufficiently salient to interfere with task-related processes. On the other hand, distractors presented outside the focus of attention will affect performance only to the extent that these stimuli can interfere with attentional processes occurring at the target location. Thus, finding out which types of distractors affect performance when positioned in an unattended location is crucial for understanding the nature of the attentional mechanism.

It is well established that distractors carrying task-relevant information (henceforth, task-relevant distractors) affect performance whether they are inside or outside the attentional focus. In the classic Stroop (1935) paradigm, for example, words denoting names of colors affect responses in a 
color naming task, lengthening or facilitating reaction times (RTs) to target colors. Importantly, this effect is observed both when the distracting words appear within the focus of visual attention (integrated with the colors, as in the typical Stroop paradigm) and when they are positioned outside the main focus of attention (spatially separated from the colors; see, e.g., Kahneman \& Henik, 1981). Similarly, taskrelevant distractors located on either side of a central target affect responses to the target in the well studied flanker task (Cohen \& Shoup, 1997; Eriksen \& Eriksen, 1974). ${ }^{2}$

The effects of other salient distractors on attentional processes are less clear. Several studies, dating back to Moray (1959), focused on the impact of personally significant but task-irrelevant distractors (e.g., the participant's own name) on task performance. These studies produced inconclusive results (e.g., Bundesen, Kyllingsbæk, Houmann, \& Jensen, 1997; Mack \& Rock, 1998; Wolford \& Morrison, 1980). As Gronau, Cohen, and Ben-Shakhar (2003) pointed out, this state of affairs may have been caused by a lack of proper control of visual attention. Gronau et al., using a focused attention task in which participants' attention was controlled, showed that personally significant stimuli affect performance and elicit an orienting response (OR - i.e., an increase in skin conductance) when presented within but not outside the focus of attention. Indeed, the effect of personally significant distractors within the focus of attention was similar in magnitude to that of task-relevant distractors, implying that these two types of distractors are equally salient. Nevertheless, only the latter affected performance when located outside the attentional focus. These findings imply that attentional processes are not linked to or affected by all salient representations but only by those associated in some way with task demands. Gronau et al. speculated that attention may be uniquely associated with representations in transient stores (e.g., working memory) that are activated during task performance.

However, the personally significant stimuli used by Gronau et al. (2003) might not be generalized to all types of salient distractors. In particular, the saliency of one's own name is derived by semantic analysis of the stimulus. It is possible that salient changes in the physical environment that do not require high-level semantic analysis would affect performance regardless of their relevance for the task. If so, attention may also be linked to and affected by salient representations that are not associated with task demands. In the present article, we address this issue by examining whether novel stimuli affect task performance when presented both within and outside the focus of attention.

By novelty, we mean any notable change in a particular dimension of the stimulus display that occurs unexpectedly and irrespective of task demands. In our study, participants were repeatedly presented with a stimulus display, and at a critical trial some aspects of the display that are irrelevant for the task suddenly changed, creating a novel display. Note in particular that this manipulation affects the physical structure of the display. Using a combined psychophysiologicalbehavioral approach and a focused attention task similar to that used by Gronau et al. (2003), we wished to determine whether novel distractor stimuli elicit an OR and affect RT, within and outside the attentional focus.

The effect of novel stimuli as defined here has been extensively investigated in the psychophysiological literature. In those studies, novelty was typically manipulated within the focus of attention, showing that attended novel stimuli elicit an enhanced OR (see, e.g., Gati \& Ben-Shakhar, 1990; Sokolov, 1963; Zimny \& Schwabe, 1965). However, the influence of novelty on task performance (e.g., response latencies) has not been investigated. Thus, in the first experiment of the present study we examined the effects of attended novel distractors on task performance. On the basis of the OR literature, we hypothesized that attended novel distractors would elicit both enhanced ORs and longer RTs to target stimuli. In the second, critical experiment, we examined whether or not similar results would be obtained when novel distractors are presented outside the main focus of attention. Finally, in the third experiment we used the same display as in the second experiment but made the novel distractors relevant for the task. By this manipulation, we sought to demonstrate that unattended task-relevant distractors are fundamentally different from unattended novel distractors.

\section{EXPERIMENT 1}

In Experiment 1, we examined the effect of attended but task-irrelevant novel stimuli. Participants named the color of a centrally presented letter. The color of the letter changed frequently between trials, but its identity was kept constant for a series of trials, after which it was unexpectedly replaced by a different letter. We assumed that the letter was within the focus of attention because it was integrated with the color target, and attention normally operates on whole objects rather than on specific features (see, e.g., Duncan, 1984).

\section{Method}

Participants. Thirty-two undergraduate students with normal or corrected-to-normal vision participated in the experiment for either course credit or payment.

Apparatus. All stimuli were presented on a color monitor connected to a Pentium computer. The participants responded vocally, and their responses were recorded by a small microphone attached to the collars of their shirts and connected to the computer. ORs were assessed by skin conductance responses (SCRs), measured by a constant voltage system ( $0.5 \mathrm{~V}$ ASR Atlas Researches). Two Ag/AgCl electrodes $(0.8 \mathrm{~cm}$ diam) were used with an electrode paste (Johnson \& Johnson K-Y Gel). The SCRs were defined as the maximal increase in conductance obtained $1-5 \mathrm{sec}$ after stimulus onset. The SCRs were computed using an A/D (NB-MIO-16) converter with a sampling rate of $1,000 / \mathrm{sec}$.

Stimuli and Design. The participants named the color of a centrally presented letter as fast as possible. The letter appeared in one of three colors: red, blue, and green. The background was dark. The size of the letter was $0.8 \mathrm{~cm}$ in height by approximately $0.7 \mathrm{~cm}$ in width, corresponding to a visual angle of approximately $0.6^{\circ} \times 0.5^{\circ}$ (from a viewing distance of $80 \mathrm{~cm}$ ).

The letter was presented repeatedly in a sequence of trials (the baseline condition), after which it was unexpectedly replaced by a different letter (the novel condition). Following this change, the baseline letter was presented for another series of trials and then 
was once again replaced by the novel letter. The experiment consisted of three blocks, each including a buffer, 21 baseline stimuli, and 2 novel stimuli. The 1 st novel stimulus in each block appeared on either the 11 th or the 12 th trial, and the 2 nd novel stimulus appeared on the 23rd or the 24th trial. For half of the participants, the baseline letter was " $X$ " and the novel letter was " $O$," and for the other half these assignments were reversed. The colors of the stimuli were determined randomly, with the constraint that the 2 novel stimuli in each block were of different colors. The experiment was preceded by a short practice block of 6 baseline trials.

Procedure. The participants were seated at a table facing the screen. After the practice block, the participants were asked to sit at ease for a period of $2 \mathrm{~min}$ while SCRs were recorded. Following this rest period, the stimulus sequence was presented. Each trial began with the presentation of a white fixation point for $500 \mathrm{msec}$, followed by presentation of a colored letter for $100 \mathrm{msec}$, followed by a white pattern mask (\#\#\#\#) covering the letter for an additional $100 \mathrm{msec}$.

The use of SCRs necessitated an unusually long intertrial interval (ITI) to allow for a recovery of the skin conductance to baseline level. Therefore, the intertrial interval in this experiment ranged from 16 to $24 \sec (M=20)$. The experiment lasted approximately $35 \mathrm{~min}$.

\section{Results and Discussion}

Trials on which the participants made errors in the color-naming task or on which RTs were below $200 \mathrm{msec}$ or above $1,500 \mathrm{msec}(<3 \%)$ were discarded from the calculations. In addition, trials on which the participants made notable movements $(2 \%)$ were excluded from the SCR analyses. In this and the remaining experiments, the proportion of errors was small and there was no hint of a possible speed-accuracy trade-off (see Table 1). Therefore, in all subsequent analyses we focused only on the RT and the OR. Table 1 presents mean RTs and ORs (i.e., SCRs) for the novel and baseline conditions of Experiment 1 , along with the effect size estimates, defined as the standardized mean differences between the novel and the baseline conditions.

As can be seen in Table 1, the novel and baseline conditions differed in both RT and OR measures. These differences were statistically significant for both measures $[t(31)=5.03, p<.001$, and $t(31)=2.40, p<.05$, respectively]. These results demonstrate that, as hypothesized, the appearance of an irrelevant novel stimulus inside the focus of visual attention impairs performance and elicits an OR. ${ }^{3}$

\section{EXPERIMENT 2}

In this experiment, we used a color naming task similar to the one used in Experiment 1, except that the distract-

Table 1

Mean Reaction Times (RTs, in Milliseconds), Mean Orienting Responses (ORs), and Error Rates (ERs), Along With Effect Size Estimates for the Novel and Baseline Conditions in the Color Naming Task of Experiment 1

\begin{tabular}{|c|c|c|c|c|c|c|}
\hline \multirow[b]{2}{*}{ Condition } & \multicolumn{2}{|c|}{ RT } & \multicolumn{2}{|c|}{ OR } & \multicolumn{2}{|c|}{ ER } \\
\hline & $M$ & $S D$ & $M$ & $S D$ & $M$ & $S D$ \\
\hline Novel & 690 & 100 & 1.66 & 1.36 & .00 & .00 \\
\hline Baseline & 646 & 85 & 1.37 & 1.13 & .01 & .01 \\
\hline Effect size estimate & 0.89 & & 0.42 & & & \\
\hline
\end{tabular}

ing letter was spatially separated from the target colors. On each trial, a central square was presented and the participants were required to name its color. Two identical white letters were presented simultaneously, one above the color square and one below it. The letters were either novel or repeated (i.e., baseline), as in Experiment 1. The goal of the experiment was to explore whether or not the novel stimuli affected RT and OR when presented outside the attentional focus.

\section{Method}

Participants. Thirty-two undergraduate students from the same population as those in Experiment 1 participated in the experiment.

Stimuli and Design. All aspects of Experiment 2 were identical to those of Experiment 1 except for the display, which included a central square and two identical letters above and below it. The size of the square was $0.8 \times 0.8 \mathrm{~cm}$ (corresponding to a visual angle of approximately $0.6^{\circ} \times 0.6^{\circ}$ ), and the size of the letters was identical to that of Experiment 1 . The center-to-center distance between the square and each of the letters was $1.6 \mathrm{~cm}\left(1.1^{\circ}\right.$ of visual angle). The participants were instructed to respond as fast as possible to the color of the square and to ignore the surrounding letters.

\section{Results and Discussion}

Extreme RTs and erroneous trials $(<2 \%)$, defined as in Experiment 1, were discarded. Trials on which the participants made notable movements $(2 \%)$ were excluded from the SCR analyses. The results for the novel and baseline conditions, along with the effect size estimates, are presented in Table 2 .

As can be clearly seen, the novelty effect obtained in the previous experiment was entirely eliminated in both measures. (In fact, there were nonsignificant effects in the opposite direction - see effect size estimates). These findings suggest that, as was the case for personally significant stimuli (Gronau et al., 2003), novel stimuli presented in an unattended region neither elicit an OR nor affect performance.

To further examine this conclusion, we performed additional statistical analyses across Experiments 1 and 2. A 2 (experiment) $\times 2$ (stimulus: novel vs. baseline) mixed ANOVA was performed on each measure (RT and OR). These analyses showed statistically significant interactions between the two factors $[F(1,62)=23.82, p<.001$ for RT, $F(1,62)=4.95, p<.05$ for OR]. These results demonstrate that, although novel stimuli affect task performance when attended, they are effectively ignored when they are presented in an unattended location.

As was previously mentioned, we hypothesized that task-relevant distractors are unique in their ability to affect attentional processes when presented at an unattended location. The goal of Experiment 3 was to dissociate the effects of task relevance and those of novelty. To this end, novel distractors were presented outside the focus of attention, as in Experiment 2, but the response requirements of the task were changed in such a way that these distractors now became relevant for the task. We hypothesized that, in contrast to the results of Experiment 2, the distractors in Experiment 3 would affect performance because they carried information associated with task requirements. Such 
Table 2

Mean Reaction Times (RTs, in Milliseconds), Mean Orienting Responses (ORs), and Error Rates (ERs), Along With Effect Size Estimates for the Novel and Baseline Conditions in the Color Naming Task of Experiment 2

\begin{tabular}{|c|c|c|c|c|c|c|}
\hline \multirow[b]{2}{*}{ Condition } & \multicolumn{2}{|c|}{ RT } & \multicolumn{2}{|c|}{ OR } & \multicolumn{2}{|c|}{ ER } \\
\hline & $M$ & $S D$ & $M$ & $S D$ & $M$ & $S D$ \\
\hline Novel & 613 & 98 & 1.16 & 1.13 & .03 & .01 \\
\hline Baseline & 624 & 81 & 1.19 & 1.06 & .01 & .01 \\
\hline Effect size estimate & -0.20 & & -0.06 & & & \\
\hline
\end{tabular}

findings would create a clear dissociation between novel (but task-irrelevant) and task-relevant distractors.

\section{EXPERIMENT 3}

\section{Method}

Participants. Thirty-two undergraduate students from the same population as those in the previous experiments participated in this experiment.

Stimuli and Design. As in Experiment 2, the display in this experiment consisted of a colored square flanked by two identical achromatic letters. However, the response requirements were changed so that, instead of being asked to name the colors, the participants were instructed to say " $\mathrm{X}$ " in response to one color (e.g., a red square), say " $Y$ " in response to a second color (e.g., a blue square), and say " $Z$ " in response to a third color (e.g., a green square). As in Experiment 2, the participants were told that any flanking letters above or below the central square were irrelevant for the task. In the baseline condition, the colored square was always flanked by the letter "O" whereas the letter " $X$ " was used for the novel flankers. However, because the participants had to utter " $X$ " in response to the red square, this letter became task relevant, creating two conditions: In the congruent trials, the novel distractor matched the required response (i.e., the letter " $X$ " was coupled with a central red square requiring an " $\mathrm{X}$ " response). In the incongruent trials, the novel distractor did not match the required response (i.e., the letter " $X$ " was coupled with either a blue or a green square requiring an alternate response). As in Experiment 2, there was a total of six novel stimuli in six trials across the three blocks of the experiment. Two of these trials were congruent, and the other four were incongruent (two with the blue square and two with the green square). The remaining trials corresponded to the baseline condition, with the letter "O" flanking the colored square. We hypothesized that a congruency effect (i.e., longer latencies for the incongruent novel trials than for the congruent novel trials) would be obtained - that is, that the unattended novel distractor that did not affect performance when it was irrelevant for the task (Experiment 2) would affect performance when it became task relevant, demonstrating a dissociation between novel and taskrelevant distractors.

To avoid possible confounding effects of specific letters on the responses to colors, the assignments of particular letters (X, Y, and Z) to specific colors (red, green, and blue) was determined randomly and counterbalanced across participants.

The experiment began with three practice blocks, which were identical to the experimental blocks. However, because OR was not measured during practice, the stimuli were presented at a relatively fast rate (every $3 \mathrm{sec}$ ). In all other aspects, the experimental procedure was identical to that employed in Experiment 2, and it lasted approximately $50 \mathrm{~min}$.

\section{Results and Discussion}

Extreme RTs and erroneous trials (4\%) were discarded as in the previous experiments. Trials on which the participants made notable movements ( $1 \%$ ) were excluded from the SCR analyses. The means of the RTs and ORs and the proportion of errors for the novel incongruent, baseline, and novel congruent conditions are presented in Table 3 . The effect size estimates, also presented in Table 3 , were defined in this experiment as the standardized mean differences between the novel incongruent and novel congruent conditions.

As can be seen in Table 3, there was a sizable congruency effect in the RT measure, reflected by slower responses to the novel incongruent stimuli than for the baseline and novel congruent stimuli. A one-way ANOVA conducted on the RT data revealed a statistically significant effect $[F(2,62)=18.13, p<.001]$. In addition, a planned comparison between the two novel conditions revealed a statistically significant difference $[t(31)=4.49, p<.001]$. This congruency effect, however, was not reflected by the OR measure, implying that OR and RT are affected by different factors. This finding converges with previous results in which task-relevant stimuli affected RT but not OR (Gronau et al., 2003). We return to this dissociation between RT and OR in the General Discussion section.

Most importantly, our findings suggest that there is a clear dissociation among novel, task-irrelevant, and taskrelevant distractors. Whereas peripheral novel stimuli had no effect on behavioral and autonomic responses in Experiment 2, the same stimuli showed a strong congruency effect when they were associated with responses to the targets in Experiment 3. Thus, although novel distractors seem to be efficiently filtered and effectively ignored, they clearly affect performance when they are associated with information relevant for task demands.

\section{GENERAL DISCUSSION}

In the present research, we compared the effects of novel distractors within and outside of the attentional focus. Two straightforward conclusions can be drawn from the present findings: First, novel stimuli influence behavioral and autonomic responses only when presented inside the focus of attention. Second, there is a fundamental difference between novel distractors and task-relevant distractors presented outside the central focus of attention. Under exactly the same conditions, the former do not affect performance whereas the latter exert a strong effect. We now elaborate on these conclusions.

Table 3

Mean Reaction Times (RTs, in Milliseconds), Mean Orienting Responses (ORs), and Error Rates (ERs) for the Novel Incongruent, Baseline, and Novel Congruent Conditions, Along With Effect Size Estimates for Differences Between the Novel Incongruent and Novel Congruent Conditions

\begin{tabular}{|c|c|c|c|c|c|c|}
\hline \multirow[b]{2}{*}{ Condition } & \multicolumn{2}{|c|}{ RT } & \multicolumn{2}{|c|}{ OR } & \multicolumn{2}{|c|}{ ER } \\
\hline & $M$ & $S D$ & $M$ & $S D$ & $M$ & $S D$ \\
\hline Novel incongruent & 804 & 121 & 0.94 & 0.99 & .02 & .07 \\
\hline Baseline & 776 & 104 & 0.98 & 1.02 & .02 & .02 \\
\hline Novel congruent & 711 & 147 & 0.98 & 1.23 & .00 & .00 \\
\hline Effect size estimate & 0.79 & & 0.06 & & & \\
\hline
\end{tabular}


The finding that attended novel distractors affect both RT and OR is in accord with previous studies (e.g., Gati \& Ben-Shakhar, 1990; Sokolov, 1963). It suggests that novel distractors are sufficiently salient to interfere with cognitive processes related to task performance, yet they do not exert any effect on behavior and do not elicit an OR when they appear in an unattended location. This finding suggests that, although salient, these stimuli do not alter processes occurring at attended locations when they are positioned elsewhere.

However, task-relevant distractors seem to play a fundamentally different role. Experiment 3 showed that novel distractors that were relevant for response-selection processes affected performance profoundly. Task-relevant distractors, then, appear to be unique in their ability to influence behavior, even when they appear in an unattended region.

As was mentioned previously, personally significant distractors (e.g., one's own name) displayed a similar pattern of results to that of novel distractors, influencing both behavioral and autonomic responses when attended but showing no effect when presented peripherally (Gronau et al., 2003). It appears, then, that stimulus saliency per se, regardless of whether it is based on semantic content (e.g., significance) or on physical features (e.g., novelty), is not sufficient to affect visual attentional processes. Task relevance, in contrast, is a powerful factor for biasing stimulus selection. Task demand representations are presumably stored in transient stores such as working memory. Thus, our findings suggest that visual attention is specifically linked to, and consequently affected by, representations that are associated with such transient stores.

On the surface, our conclusions seem to be related to claims made by Folk, Remington, and Johnston (1992) concerning the unique ability of stimuli that have some relation to attentional settings (i.e., task-relevant stimuli) to capture visual attention. However, these investigators examined mostly search tasks in which attention is not necessarily focused at any specific region. Presumably, in such situations attention is "on the move" and thus might be more prone to capture. Indeed, several investigators have suggested that attention can be captured even by some exogenous, task-irrelevant stimuli in visual search tasks (e.g., Theeuwes \& Godijn, 2001; Yantis \& Jonides, 1984). In the present study, we used a focused attention paradigm in which task demands require the participant to focus attention on a prespecified location and to avoid attentional shifts. Thus, although the patterns of results in the two domains appear similar, it is not clear whether the same mechanisms are at work. Future research is required to clarify this issue.

Finally, the present results shed further light on the relation between RT and OR measures. Whereas these measures displayed a similar pattern in Experiments 1 and 2, the congruency effect in Experiment 3 was evident only in the RT measure. Similarly, Gronau et al. (2003) showed in several experiments that task-relevant distractors affect primarily RT and exert only a small (and often insignificant) effect on OR. These findings suggest that OR is not elicited by all types of salient stimuli. Rather, it may be elicited by specific types of stimuli, such as those arising from attended but irrelevant salient distractors. Uncovering the specific factors involved in OR elicitation is an important goal for future research.

\section{REFERENCES}

Bundesen, C., Kyllingsbæk, S., Houmann, K. J., \& Jensen, R. M. (1997). Is visual attention automatically attracted by one's own name? Perception \& Psychophysics, 59, 714-720.

Cohen, A., \& Shoup, R. (1997). Perceptual dimensional constraints in response selection processes. Cognitive Psychology, 32, 128-181.

Duncan, J. (1984). Selective attention and the organization of information. Journal of Experimental Psychology: General, 113, 501-517.

ERIKSEN, B. A., \& ERIKSEN, C. W. (1974). Effects of noise letters upon the identification of a target letter in a nonsearch task. Perception \& Psychophysics, 16, 143-149.

Folk, C. L., Remington, R. W., \& Johnston, J. C. (1992). Involuntary covert orienting is contingent on attentional control settings. Journal of Experimental Psychology: Human Perception \& Performance, 18, 1030-1044.

Gati, I., \& Ben-Shakhar, G. (1990). Novelty and significance in orientation and habituation: A feature-matching approach. Journal of Experimental Psychology: General, 119, 251-263.

Gronau, N., Cohen, A., \& Ben-Shakhar, G. (2003). Dissociations of personally significant and task-relevant distractors inside and outside the focus of attention: A combined behavioral and psychophysiological study. Journal of Experimental Psychology: General, 132, 512-529.

Kahneman, D., \& Henik, A. (1981). Perceptual organization and attention. In M. Kubovy \& J. R. Pomerantz (Eds.), Perceptual organization (pp. 181-211). Hillsdale, NJ: Erlbaum.

LaBerge, D. (1983). Spatial extent of attention to letters and words. Journal of Experimental Psychology: Human Perception \& Performance, 9, 371-379.

MACK, A., \& Rock, I. (1998). Inattentional blindness. Cambridge, MA: MIT Press.

Moray, N. (1959). Attention in dichotic listening: Affective cues and the influence of instructions. Quarterly Journal of Experimental Psychology, 11, 56-60.

Posner, M. I. (1980). Orienting of attention. Quarterly Journal of Psychology, 32, 3-25.

RAuschenberger, R. (2003). Attentional capture by auto- and allocues. Psychonomic Bulletin \& Review, 10, 814-842.

Sokolov, E. N. (1963). Perception and the conditioned reflex (S. W. Waydenfeld, Trans.). New York: Macmillan.

Stroop, J. R. (1935). Studies of interference in serial verbal reactions. Journal of Experimental Psychology, 18, 643-662.

Theeuwes, J., \& GodiJn, R. (2001). Attentional and oculomotor capture. In C. L. Folk \& B. S. Gibson (Eds.), Attraction, distraction and action: Multiple perspectives on attentional capture (Advances in Psychology, Vol. 133, pp. 121-149). New York: Elsevier.

Wolford, G., \& Morrison, F. (1980). Processing of unattended visual information. Memory \& Cognition, 8, 521-527.

YANTIS, S., \& JoNIDES, J. (1984). Abrupt visual onsets and selective attention: Evidence from visual search. Journal of Experimental Psychology: Human Perception \& Performance, 10, 601-621.

Zimny, G. H., \& Schwabe, L. W. (1965). Stimulus change and habituation of the orienting response. Psychophysiology, 2, 103-114.

\section{NOTES}

1. The present study concerns focused attention tasks. Many other studies (see Rauschenberger, 2003, for a review) have been conducted to examine the effects of unattended distractors in visual search tasks in which the location of the target is not known in advance. The attentional demands in these two types of paradigms are different. As is discussed more extensively in the General Discussion section, it is not clear whether the effect of distractors in the two paradigms tap similar mechanisms. Therefore, we restrict our review to focused attention tasks. 
2. The term task relevance is sometimes used in a narrow sense and pertains only to stimuli or features that are actually relevant for the task. Here, we define task relevance in a broader sense, as pertaining to any information that is associated with the attentional setting of the participant while he or she is performing the task (see e.g., Folk, Remington, \& Johnston, 1992, for a similar approach). For example, the content of the distractor words in the classical Stroop task is not relevant for the task in the narrow sense, but it is relevant in the broader sense (when it denotes a color), since participants respond to colors (see also Gronau, Cohen, \& Ben-Shakhar, 2003).

3. Note that the letter in most baseline trials did not differ from the letter in the immediately preceding trials, whereas the letter in the novel trials did differ from that in the immediately preceding trials. One may argue that it is the change between successive trials in the novel condi- tion, rather than novelty per se, that might have caused the longer RTs and higher ORs. According to this alternative interpretation, the trials immediately following the novel trials should also show the same effect because the letter in those trials also changed (from the novel to the baseline letter). This prediction was not supported by the data. The mean RT in these trials was $637 \mathrm{msec}$, slightly shorter than the mean for the baseline condition $(646 \mathrm{msec})$. Likewise, the mean SCR in these trials was 1.31 , slightly lower than the mean SCR for the baseline condition (1.37). This analysis clearly demonstrates that the effect we obtained in Experiment 1 is due to novelty rather than to the change between successive trial types.

(Manuscript received May 27, 2005;

revision accepted for publication December 16, 2005.) 